Signal \& Image Processing : An International Journal (SIPIJ) Vol.4, No.4, August 2013

\title{
ANALYSIS OF BRAIN COGNITIVE STATE FOR ARITHMETIC TASK AND MOTOR TASK USING ELECTROENCEPHALOGRAPHY SIGNAL
}

\author{
R Kalpana ${ }^{1}$, M Chitra $^{2}$, Navkiran Kalsi ${ }^{3}$, Rajanikant Panda ${ }^{4}$ \\ ${ }^{1}$ Dept. of Medical Electronics, B.M.S college of Engineering, Bangalore, India \\ ${ }^{2}$ Dept. of Information Science and Technology, \\ Sona College of Technology, Salem, India \\ ${ }^{3}$ Centre for Converging Technologies, University of Rajasthan, Jaipur, India \\ ${ }^{4}$ Dept. of Bio-Medical Engineering, \\ Trident Academy of Technology, Bhubaneswar, India \\ kalpana4researchegmail. com
}

\begin{abstract}
To localize the brain dynamics for cognitive processes from EEG signature has been a challenging task from last two decades. In this paper we explore the spatial-temporal correlations of brain electrical neuronal activity for cognitive task such as Arithmetic and Motor Task using $3 D$ cortical distribution method. Ten healthy right handed volunteers participated in the experiment. EEG signal was acquired during resting state with eyes open and eyes closed; performing motor task and arithmetic calculations. The signal was then computed for three dimensional cortical distributions on realistic head model with MNI152 template using standardized low resolution brain electromagnetic tomography (sLORETA). This was followed by an appropriate standardization of the current density, producing images of electric neuronal activity without localization bias. Neuronal generators responsible for cognitive state such as Arithmetic Task and Motor Task were localized. The result was correlated with the previous neuroimaging (fMRI study) investigation. Hence our result directed that the neuronal activity from EEG signal can be demonstrated in cortical level with good spatial resolution. $3 D$ cortical distribution method, thus, may be used to obtain both spatial and temporal information from EEG signal and may prove to be a significant technique to investigate the cognitive functions in mental health and brain dysfunctions. Also, it may be helpful for brain/human computer interfacing.
\end{abstract}

\section{KEYWORDS}

EEG, sLORETA, Arithmetic Task, Motor Task, MNI152

\section{INTRODUCTION}

From past two decades to understand the brain for neuro-cognitive processes is the foremost query to the cognitive neuroscience researchers. This is because of two main reasons: one, for brain activity analysis that reveals how different brain regions interact with each other and second, the current necessity to understand how our brains impact our perception of our environment and how our brain function affects our behavior.

DOI : 10.5121/sipij.2013.4404 
Signal \& Image Processing : An International Journal (SIPIJ) Vol.4, No.4, August 2013

Electroencephalography (EEG) has an amazing capability to investigate the brain networks and classify the brain state for cognitive function [1], [4], [6], [7], [8] and thus has been extensively used for research and clinical purposes. EEG analyses for Cognitive Functions show us "how" our brain is functioning on neuronal level with macroscopic scale [2], [5]. From previous studies it is noted that the EEG can examine brain function with high temporal resolution but it has some limitation- it doesn't contain sufficient information on the three-dimensional (3D) distribution of electric neuronal activity. On contrary, functional imaging techniques such as Positron emission tomography (PET) and functional magnetic resonance imaging provide three-dimensional (3D) images with an excellent spatial resolution, the temporal resolution is not high enough to match with the speed at which neuronal processes occur. To solve the paradox, a growing number of studies have been published that make use of functional imaging methods based on the electroencephalogram (EEG) and the magnetoencephalogram (MEG) [1], [11]. The methods proposed include quantitative spectral EEG analysis (qEEG), different computational algorithms such as fast Fourier transform (FFT) or auto regressive (AR) models (Brenner et al., 1986; Coben et al., 1985; Giaquinto and Nolfe, 1986; Prinz et al., 1992) Independent component analysis, time-frequency (wavelet) analysis. But still many debate and query have there on brain dynamics measurement for cognitive task using EEG signals.

In this paper, we examine the scalp EEG signal with spatial-temporal correlations for resting state brain and cognitive state using 3D cortical distribution method. Ten healthy right handed participants were taken for Electroencephalography (EEG) signal recording in resting eye open and resting eye close (non-cognitive) and also while performing cognitive tasks such as motor sensory task and arithmetic calculation task. The EEG signal was computed for three dimensional cortical distributions on realistic head model using the MNI152 template using standardized low resolution brain electromagnetic tomography (sLORETA) [9], [10], 14]. The brain networks were examined with scalp and cortical level and the result was compared with the previous neuro imaging research such as functional Magnetic Resonance Imaging (fMRI) study.

\section{MATERIAL AND METHODS}

\subsection{Subjects Details}

Ten adult right-handed healthy people with a mean age of 28 years (SD \pm 5 years) participate for electroencephalography (EEG) signal recording. The participants had no history of neurological or psychiatric disease and did not take any medication that could affect the experiment. All participants have more than five teen years of education and with IT (Information Technology) professional skill.

\subsection{EEG Data Acquisitions}

The EEG cap consisted of 31 uni polar scalp electrodes placed according to the international 10-20 system electrode placement and one additional electrode dedicated to the vertical electrooculogram (EOG) refer fig 1. Data were recorded relative to an FCz reference and a ground electrode was located at Iz (10-5 electrode system, (ostenveld and Praamstra, 2001). Data were sampled at 1000 $\mathrm{Hz}$ and the impedance between electrode and scalp was kept below $5 \mathrm{k} \Omega$. Data was acquired in a close room with a comfort sit. The room was containing very minimum no of electronic gadget and very good grounding. The paradigm instruction was given via small mike situated inside the room. Inside the room one LCD monitor has there for visual representation. 


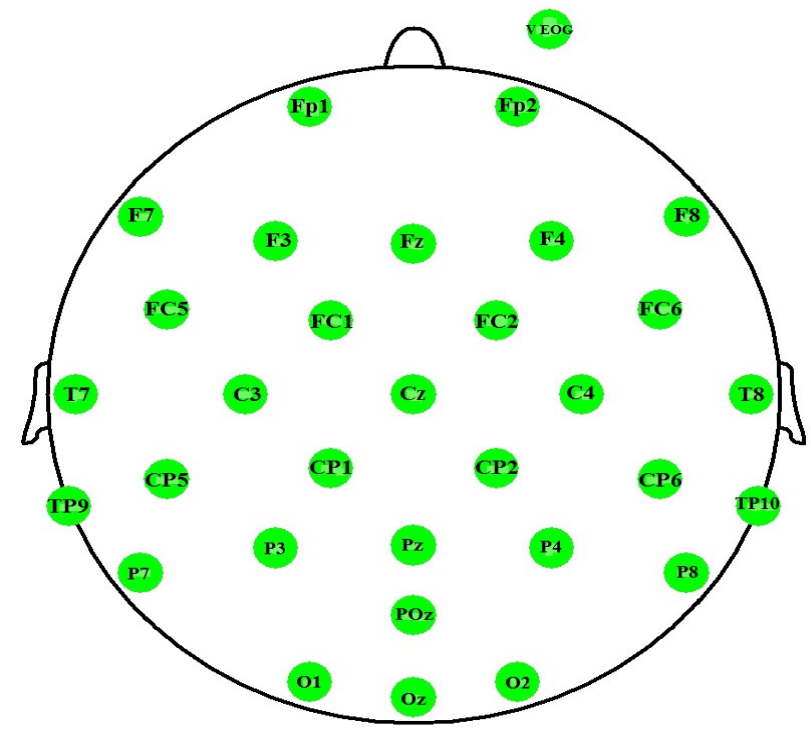

Fig 1. International 10-20 system for electrode placement

\subsection{Paradigm for Different Tasks}

Three type of cognitive task was performed fallowed to eye open and eye close at relax resting condition. Details are fallowed.

\subsubsection{Instruction for eye open, close at rest}

Subject was instructed to be sited with relax condition, as per instruction subjects was open their eye for five minute and closed their eye for five minute. At that time instruction was given to do not do any cognitive task like language, attention, memory related or motor tasks as much as possible.

\subsubsection{Instruction for Motor task}

Subject was instructed four sections such as Relax, to squeeze right hand, relax, to squeeze left hand. Each of section was thirty second and whole cycle was repeated three times.

\subsubsection{Instruction for Arithmetic Calculation}

Subject was instructed to count the Fibonacci sequence is the series of numbers: $0,1,1,2,3,5,8$, $13,21,34 \ldots$ from 1 to 100 .

\section{DAta Analysis}

\subsection{Pre Processing and Artifact Removal from EEG signal}

Raw EEG data were processed offline using MATLAB software. The Data was down-sampled to $250 \mathrm{~Hz}$. After that 4th order band-pass Butterworth FIR filter was applied with a cut-off frequency of 0.01 to $60 \mathrm{~Hz}$ for attenuate low frequency noise (DC offset voltage, movement artifact) \& high 
Signal \& Image Processing : An International Journal (SIPIJ) Vol.4, No.4, August 2013

frequency noise (environmental noise, instrumental noise), then $50 \mathrm{~Hz}$ notch filter was applied for power line interference [3], [8]. After that baseline correction was done. Once artifacts had been removed, the data were then inspected visually for artifacts resulting from muscular sources or any other electro-physiological artifact and if any epochs containing voltage of more than $150 \mu \mathrm{V}$ was manually rejected. After that the EEG data was taken for further study.

\subsection{Post Processing for EEG Signal}

On the basis of the scalp-recorded electric potential distribution, sLORETA (tomographic method) was used to compute the cortical three-dimensional distribution of current density for all segmented data sets (Resting Eye open, resting Eye close, motor task, attention and memory task). Low resolution electromagnetic tomography (LORETA) assumes that the smoothest of all activity distributions is most plausible ("smoothness assumption") and therefore, a particular current density (d) distribution is found [9]. This method followed by an appropriate standardization of the current density, producing images of electric neuronal activity without localization bias. Computations were made in a realistic head model using the MNI152 template [10] with the threedimensional solution space restricted to cortical gray matter. sLORETA images represent the electric activity at each voxel in neuro anatomic Talairach space as the squared standardized magnitude of the estimated current density.

To overcome the EEG inverse problem, a method is the dipole source with fixed locations and orientations that are distributed in the whole brain volume or cortical surface. There are six parameters that specify the dipole, three spatial coordinates $(\mathrm{x}, \mathrm{y}, \mathrm{z})$ and three dipole moment components (orientation angles $(\theta, \varphi)$ and strength $\mathrm{d}$ ). The sources are intracellular currents in the dendritic trunks of the cortical pyramidal neurons, which are normally oriented to the cortical surface [6], fixed orientation dipoles are generally set to be normally aligned. The amplitudes (and direction) of these dipole sources are then estimated. Since the dipole location is not estimated the problem is a linear one.

There are $\mathrm{N}_{\mathrm{E}}$ instantaneous extra cranial measurements in the surface of brain and $\mathrm{N}_{\mathrm{V}}$ voxels in the brain. Typically, the voxels are determined by subdividing uniformly the solution space, which is usually taken as the cortical grey matter volume or surface. At each voxel there is a point source, which may be a vector with three unknown components (i.e., the three dipole moments), or a scalar (unknown dipole amplitude, known orientation). The cases considered here correspond to $\mathrm{N}_{\mathrm{V}}>>\mathrm{N}_{\mathrm{E}}$.

The Bayesian methods find an estimate $\mathrm{D}$ is

$$
\mathrm{D}=\min (\mathrm{U}(\mathrm{d})) ; \quad \text { where } \mathrm{U}(\mathrm{d})=\|\mathrm{M}-\mathrm{Gd}\|_{\mathrm{R}}{ }_{\mathrm{R}}+\alpha L(\mathrm{~d})
$$

$\mathrm{G}=$ Generalized Cross Validation, $\mathrm{M}$ is the matrix of data measurements at different times $\mathrm{m}(\mathrm{r}, \mathrm{t})$ and $\mathrm{d}$ is the matrix of dipole moments at different time instants.

The sLORETA uses the current density estimate given by the minimum norm estimate $D_{\text {MEN }}$ (MNE-Minimum norm estimates) and standardizes it by using its variance, which is hypothesized to be due to the actual source variance $S_{D}=I_{3 p}$, and variation due to noisy measurements $S^{M}{ }_{n o i s e}=$ $\alpha \mathrm{I}_{\mathrm{N}}$. The electrical potential variance $\mathrm{S}_{\mathrm{M}}=\mathrm{GS}_{\mathrm{D}} \mathrm{G}^{T}+\mathrm{S}^{\mathrm{M}}$ noise and the variance of the estimated current density is $S_{D}=T_{M N E} S_{M} T^{T}{ }_{M N E}=G^{T}\left[G G^{T}+\alpha I_{N}\right]^{-1} G$. This is equivalent to the resolution matrix $\mathrm{T}_{\mathrm{MNE}} \mathrm{G}$. For the case of EEG with unknown current density vector, sLORETA gives the following estimate of standardized current density power: 
Signal \& Image Processing : An International Journal (SIPIJ) Vol.4, No.4, August 2013

$$
\mathrm{D}^{\mathrm{T}}{ }_{\mathrm{MNE}, 1}\left\{\left[\mathrm{~S}_{\mathrm{D}}\right]_{l l}\right\}^{-1} \mathrm{D}_{\mathrm{MNE}, 1}
$$

where $\mathrm{D}_{\mathrm{MNE}, 1} \in \mathrm{R}^{3 \times 1}$ is the current density estimate at the $l$ th voxel given by the minimum norm estimate and $\left[\mathrm{S}_{\mathrm{D}}\right]_{l l} \in \mathrm{R}^{3 \times 3}$ is the $l$ th diagonal block of the resolution matrix $\mathrm{S}_{\mathrm{D}}$. It was found [16] that in all noise free simulations, although the image was blurred, sLORETA had exact, zero error localization when reconstructing single sources, that is, the maximum of the current density power estimate coincided with the exact dipole location. In all noisy simulations, it had the lowest localization errors when compared with the minimum norm solution and the Dale method [17]. The Dale method is similar to the sLORETA method in that the current density estimate given by the minimum norm solution is used and source localization is based on standardized values of the current density estimates. However, the variance of the current density estimate is based only on the measurement noise, in contrast to sLORETA, which takes into account the actual source variance as well.

\section{RESULT}

\subsection{Resting state With Eye Open and Eye Close Condition}

Resting state With Eye Open and Eye Close Condition as per neuroscience when a person with relax and resting state and not involved with any physical and mental work, the activity occur in the brain may random and unpredictable. In relax eye open and eye closed condition the frequency range will be differ [13]. Awake and relax with eye open state lower beta range $(10 \mathrm{~Hz}$ to $14 \mathrm{~Hz})$ frequency will be dominated, in other hand awake and relax with eye close condition alpha range $(7 \mathrm{~Hz}$ to $11 \mathrm{~Hz})$ frequency will be dominated.

From our study we find out high variability in activated brain areas for awake and relax state in both eye open and eye close condition. And we observe mostly occipital and frontal areas activation at the time of resting state. In eye open condition we observe occipital lobe was dominate with many other brain areas.

\subsection{Complex Arithmetic Calculation Task}

Number theory is a complex achievement of the human mind. However, the core concept of arithmetic number is simple, and all human cultures have at least a few words for numbers. For complex arithmetic tasks, the brain involved multifunction such as memory, planning and executing and calculation. Also the sympathetic emotion involved at the time of complex arithmetic tasks [12], [15].

From our study we find out for complex arithmetic tasks frontal lobe such as inferior prefrontal cortex, right dorsa lateral prefrontal cortex and bilateral parietal lobe and cingulate brain areas was activated. See the figure 2 .

\subsection{Sensory Motor Task}

The sensory motor task is to understand sensation and motor activity such as movement. In this study we find out the frontal lobe and parietal lobe are activated for Sensory Motor Task. For most of the subject we got bilateral activation for both right hand and left hand motor task but 
Signal \& Image Processing : An International Journal (SIPIJ) Vol.4, No.4, August 2013

for right hand motto task we got left cerebellum dominate activation and for left hand motto task we got right cerebellum dominate activation.

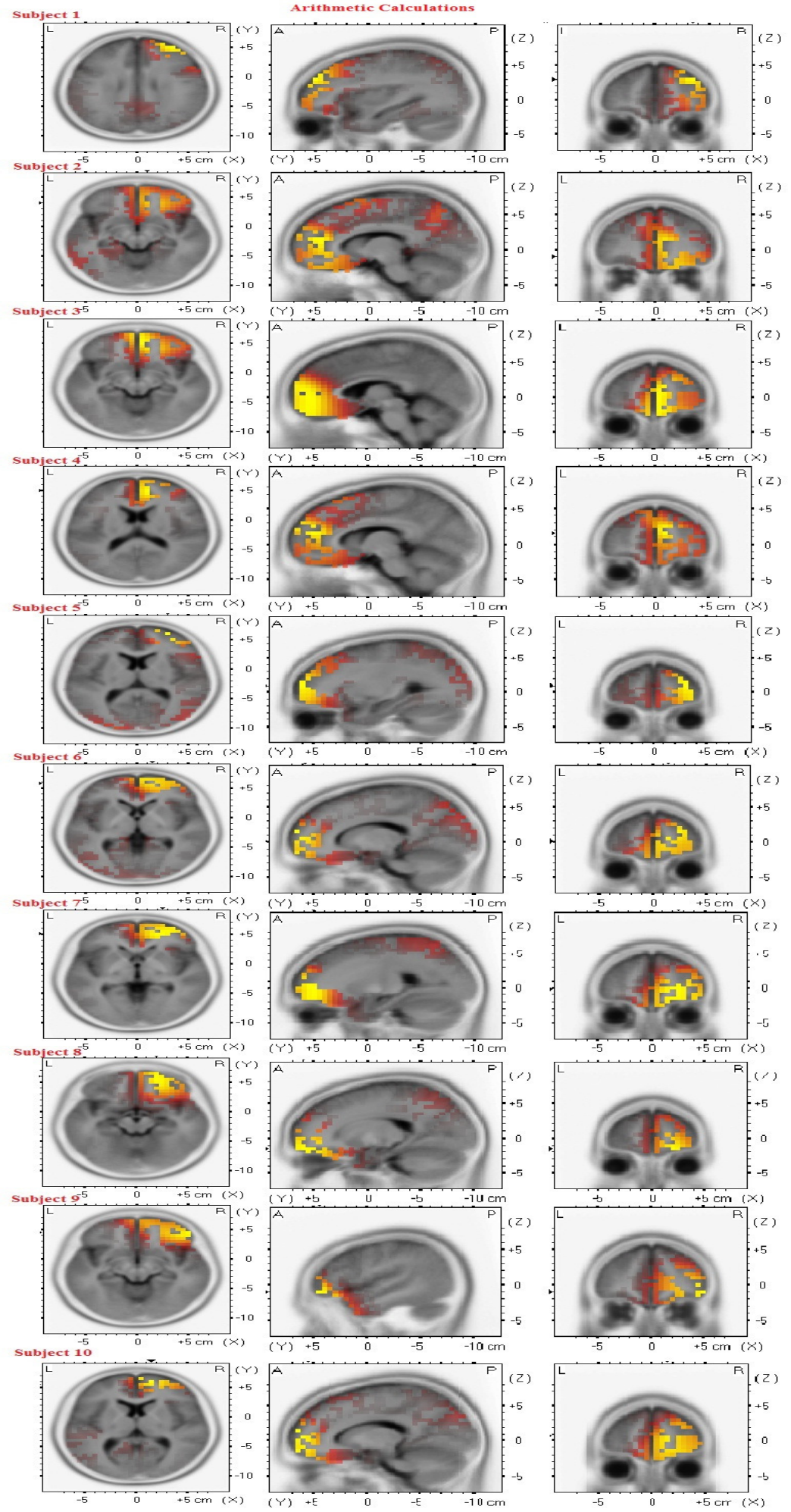

Fig 2. Neuronal activation in MNI template for complex arithmetic calculation task (Fibonacci sequence) computed from EEG signal. 
Signal \& Image Processing : An International Journal (SIPIJ) Vol.4, No.4, August 2013

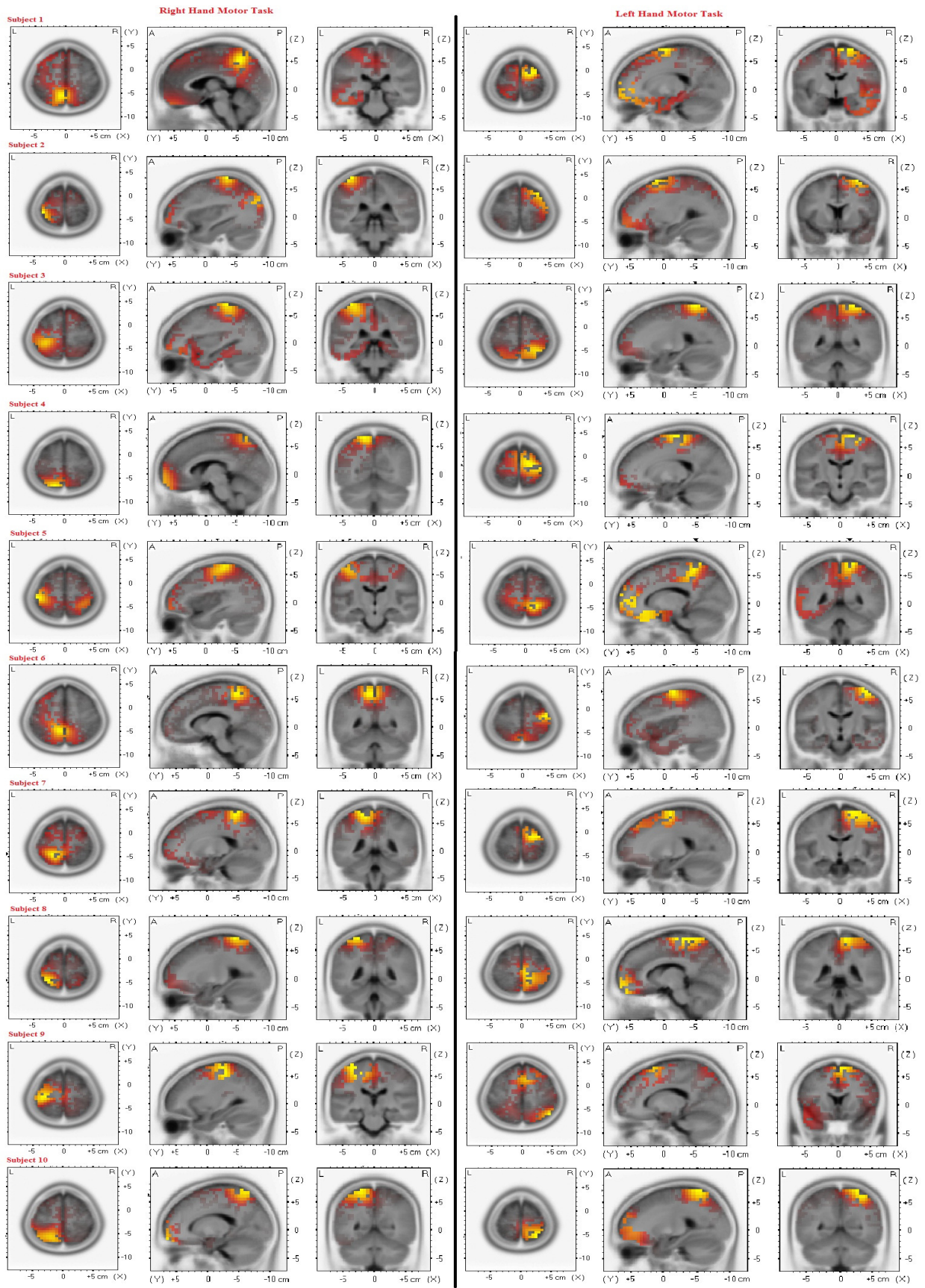

Fig 3. Neuronal activation in MNI template for right hand and left hand motor task computed from EEG signal.

\section{CONCLUSION}

To examine brain states for cognitive processes using EEG signal is a unique technique as it is direct method to measure neuronal activity but it have the limitation on spatial resolution. We examined brain networks from EEG signal using 3D cortical distribution method. This method followed by an appropriate standardization of the current density, producing images of electric neuronal activity without localization bias. Brain regions are significantly distinguished for cognitive state such as arithmetic calculation task and motor task. The result was correlating with 
Signal \& Image Processing : An International Journal (SIPIJ) Vol.4, No.4, August 2013

the previous neuroimaging research (fMRI study) investigation. Hence this result indicates that, the temporal and spatial dimensions of cortical activity from EEG signal can be demonstrated using sLORETA making it an important and affordable tool for cognitive function analysis in clinical neurosciences such as mental health, traumatic and acquired brain dysfunction or injury etc also it may helpful for brain/human computer interfacing.

\section{REFERENCES}

[1] Michel, C.M., Murray, M.M.: Towards the utilization of EEG as a brain imaging tool. NeuroImage. 61, 371-385 (2012)

[2] Anderson, C.W., Devulapalli, S.V., Stolz, E. A.: Determining Mental State from EEG Signals Using Neural Networks. Scientific Programming. 4, 171-83 (1995)

[3] Garrett, D., Peterson, D.A., Anderson, C.W., Thaut, M.H.: Comparison of Linear, Nonlinear, and Feature Selection Methods for EEG Signal Classification. IEEE transactions on neural systems and rehabilitation engineering. 11, 141- 144 (2003)

[4] Carlino, E., Sigaudo, M., Pollo, A., Benedetti, F., Mongini, T., Castagna, F. , Vighetti, S., Rocca, P.: Nonlinear analysis of electroencephalogram at rest and during cognitive tasks in patients with schizophrenia. J Psychiatry Neurosci. 37, 259-266 (2012)

[5] Dyson, M., Sepulveda, F., Gan, J.Q.: Localisation of cognitive tasks used in EEG-based BCIs. Clinical Neurophysiology. 21, 1481-1493 (2010)

[6] Evans, N.: Single-Trial analyses of novel cognitive tasks in an EEG-based brain-computer interface framework unpublished

[7] Srinivasan, N.: ognitive Neuroscience of Creativity: EEG based approache. Methods, 42, 109-116, (2007)

[8] Pal, P.R., Khobragade, P., Panda, R.: Expert system design for classification of brain waves and epileptic-seizure detection. IEEE Students' Technology Symposium (TechSym), 187-192 (2011)

[9] Pascual-Marqui, R.D.: Standardized low resolution brain electromagnetic tomography (sLORETA): technical details. Methods \& Findings in Experimental \& Clinical Pharmacology, 24, 46-72 (2002)

[10] Cannon, R.L., Thatcher, R.W., Lubar, J. F., Baldwin, D. R., Lubar, J. F., EEG LORETA and the Default Mode of the Brain. unpublished

[11] Palaniappan, R., Raveendran, P.: Cognitive Task Prediction Using Parametric Spectral Analysis of EEG Signals. Malaysian Journal of Computer Science, 14, 58-67 (2001)

[12] Dehaene, S. , Molko, N., Cohen L., Wilson A.J.: Arithmetic and the brain. Current Opinion in Neurobiology. 14, 218-224 (2004)

[13] Liu, T. ., Shi, j., Zhao, D., Yang, J.: The relationship between EEG band power, cognitive processing and intelligence in school ege children. Psychology Science quarterly, 50, 259-268 (2008)

[14] Collura, T.F.: Individualized assessment and treatment using advanced EEG and dynamic localization techniques with live sLORETA. International Journal of Psychophysiology. 85, 285-290 (2012)

[15] Menon, V., Rivera, S.M., White, C.D., Glover, G.H , Reiss, A.L: Dissociating prefrontal and parietal cortex activation during arithmetic processing. Neuroimage. 12, 357-365 (2000)

[16] Pascual-Marqui R.:, Standardized low resolution brain electromagnetic tomography (sLORETA):technical details. Methods and Findings in Experimental \& Clinical Pharmacology. 24, 5$12(2002)$

[17] Dale, A., Liu, A., Fischl, B., Buckner, R., Belliveau, J., Lewine, J., Halgren E.: Dynamic statistical parametric mapping: combining fMRI and MEG for high-resolution imaging of cortical activity" Neuron. 26, 55-67 (2000) 
Signal \& Image Processing : An International Journal (SIPIJ) Vol.4, No.4, August 2013

\section{AUTHOURS PROFILE:}

Mrs. R. KALPANA received B.E degree in Electronics and communication Engineering from Hindustan college of Engineering, University of Madras, in 1992 and received M.Tech degree in Digital Communications. from Department of Electronics, B.M.S College of Engineering, Visvesvaraya Institute of Technology in 2005, Bangalore. She has 12 years of teaching experience in field of Biomedical Electronics. Currently she is working as Asst. Prof in Department of Medical electronics. She has published 4 international research papers.

Prof. M. CHITRA received PhD Degree in Information and communication engineering from Anna university of Technology Chennai, in the year 2009. She is currently working as Prof. Department of Information science and Technology, Sona College of Technology. She has 15 years of teaching experience in field of Information science and communication Engineering. Her Area of Interest is Mathematics, Neural networks, communication and signal processing and Image processing. She has published more than 10 International research papers.

Ms. Navkiran Kalsi, received B.Tech and M.Tech degree in Cognitive Neuroscience from 3Centre for Converging Technologies, University of Rajasthan, India, in 2012. She has 1 year of research experience on Neuroimaging and EEG signal processing on cognation at National Institute of Mental Health and Neurosciences, Bangalore, India. Currently she is working in a DST project at National Institute of Mental Health and Neurosciences as a Research Fellow.

Mr. Rajanikant Panda received B.Tech degree in Biomedical Engineering from Trident Academy of Technology, Biju Patnaik University of Technology (BPUT), in 2010. He has 1 year of Bio-Medical Industrial Experience at Humankarigar Pvt. Ltd. and 2 year of research experience on multimodal Neuroimaging and EEG signal processing from National Institute of Mental Health and Neurosciences Bangalore, India. Currently he is working in a DST project at National Institute of Mental Health and Neurosciences as a Research Fellow. He has published 8 international research papers. 